\title{
Comparison of surface and bulk nitrogen modification in highly porous carbon for enhanced supercapacitors
}

\author{
Stephanie L. Candelaria, Evan Uchaker and Guozhong Cao*
}

Highly porous carbon prepared through sol-gel processing can be modified with nitrogen via a simple solution-based method to achieve increased capacitance. Nitrogen was added either in the form of a surface coating using hexamine or incorporated into the bulk carbon network upon heat treatment of the hexamine-coated carbon. The properties and electrochemical behavior of both materials were measured and compared to that of unmodified porous carbon. Nitrogen in the bulk carbon network shows increased conductivity, while nitrogen coated on the surface of porous carbon is more effective at increasing capacitance. Nitrogen modification can also reduce diffusivity resistance and charge transfer resistance, resulting in supercapacitors with better performance at higher charge and discharge rates.

\section{INTRODUCTION}

As the popularity of alternative energy increases, the need for energy storage devices increases as well. Wind, solar, and other renewable energy resources require to be collected and stored during peak generation times, while allowing easy recovery in order to meet demand during off-peak hours [1]. This calls for the use of energy storage devices, such as batteries, fuel cells, and capacitors. Ideal devices share many characteristics, including large storage capacity, fast transport kinetics, and cyclic fatigue resistance [2-7].

In electric double layer capacitors (EDLCs), which are also known as supercapacitors or ultracapacitors, charge storage takes place through the formation of an electric double-layer structure at the interface between the electrodes and electrolyte. As such, the capacitance is strongly dependent on the surface area of the electrodes. Porous carbon has become the most commonly used electrode material in EDLCs [8], because it is electrically and thermally conductive, chemically inert, mechanically strong, and has a low density [9]. Historically, research efforts have focused on achieving a higher capacitance by maximizing the surface area of the electrode material through careful thermal, chemical, or electrochemical treatment. Today, it is common for the specific surface area of activated carbon to reach well over $2000 \mathrm{~m}^{2} \mathrm{~g}^{-1}$. However, problems arise as the specific surface area increases beyond a certain value. For example, the linear relationship between capacitance and surface area exists only up to a certain point. Above $1500 \mathrm{~m}^{2} \mathrm{~g}^{-1}$, the capacitance begins to level off, no longer increasing linearly with surface area [10]. One explanation for this is that the number of small micropores $(\sim 1 \mathrm{~nm})$ increases for high surface area samples. Micropores of this size are too small to allow electrolyte ions to diffuse inside, resulting in inaccessible surface area that goes against to the charge storage $[10,11]$. Additionally, carbons with very high surface area have a very low volumetric density, and reduced mechanical and chemical stability. Therefore, it is essential to find a balance between high surface area to increase the number of sites available for charge storage and an open pore structure for fast and efficient transport of the electrolyte ions into the pores. This could be achieved through introducing hierarchical pore structures, where micropores provide high surface area and mesopores and macropores provide open porosity $[11,12]$.

Surface chemistry modification can be used in addition to optimizing surface area and pore structure to enhance performance. Intentionally doping porous carbon with foreign elements, including boron, nitrogen, sulfur, phosphorous, and others, can lead to higher surface charge density and the addition of pseudocapacitive reactions, leading to higher capacitance $[5,13-17]$. The majority of work in this area has focused on introducing oxygen heteroatoms into graphene layers of the carbon network. Often the oxygen functionalities are acidic in nature, inducing electron acceptor characteristics into the carbon materials [18]. While oxygen functionalities are beneficial in aqueous electrolytes, their presence in organic electrolytes is detrimental to the performance due to irreversible reactions between

Department of Materials Science and Engineering, University of Washington, Seattle, WA 98195-2120, USA

*Corresponding author (email: gzcao@u.washington.edu) 
oxygen and the electrolyte [19]. Organic electrolytes are of interest due to their higher voltage window, which can lead to higher energy density. Using aqueous electrolyte, the maximum device voltage is less than $1 \mathrm{~V}$ as voltages above this value approach the thermodynamic voltage window of water $(1.23 \mathrm{~V})$, at which point the electrolyte decomposes [20]. However, the voltage window for organic electrolytes is much larger. Devices are typically tested at $2 \mathrm{~V}$, with the stability window typically reaching up to $2.7 \mathrm{~V}$ [21]. To take full advantage of the much higher voltage window of organic electrolytes, it is necessary to find an alternative to oxygen that will also induce pseudocapacitive reactions.

Recently, a number of studies reported improved capacitance by using carbon enriched with nitrogen in both aqueous electrolytes [22-27] and organic electrolytes $[25,27,28]$. Many of these studies incorporate nitrogen into the bulk network as heteroatoms, either through the use of a nitrogen-containing precursor [22,29-32] or modification after carbon synthesis [18,33-37]. Two of the most common carbon-nitrogen structures found within the material include pyrrole groups with nitrogen in a 5-member ring and pyridine groups with nitrogen in a 6-member ring [38]. The presence of pyrrolic nitrogen adds two p-electrons to the delocalized $\pi$-electron system and a hydrogen atom, which is bound in the plane of the ring. Pyridinic nitrogen contributes one p-electron to the $\pi$-electron system and one lone electron pair in the plane of the ring [38]. Nitrogen also occurs as quaternary nitrogen, which is within a 6-member ring as well, but located at "center" or "valley" positions [18]. Quaternary nitrogen is slightly more positively charged, resembling quaternary nitrogen in ammonium ions $\left(\mathrm{NH}_{4}^{+}\right)$[39]. It is suggested that the quaternary nitrogen is a form of pyridinic nitrogen associated with a nearby hydroxyl or carboxyl group, which protonates the nitrogen by forming a hydrogen bridge [38]. Other researchers claim that the quaternary nitrogen has pyrrolic characteristics, increasing the electron-donor effect [18]. However, there has not yet to be a solid conclusion concerning which of these groups provides the most benefit for improving the performance of carbon-based supercapacitors.

In addition to substituting into the carbon network, nitrogen can also be added to porous carbon in the form of surface functional groups $[35,40,41]$. This is often at the relatively reactive graphene edges, where carbine and carbine-type free edge sites are present [21]. Common functional groups include amines, imines, amides, and imides $[18,21]$. Our recent report [42] concluded that nitrogen coated on the surface of the pores, rather than being incorporated into the bulk carbon network as heteroatoms, contributes the most to increasing capacitance. Despite similar nitrogen contents, samples with nitrogen on the surface outperform samples with nitrogen only in the bulk network. The surface modified samples have dramatically improved wettability and a capacitance that is nearly twice over that of the unmodified carbon, achieved through a combination of double-layer formation and pseudocapacitive reactions. However, the specific surface area was only moderate due to a relatively low pyrolysis temperature and a lack of activation to improve the porous structure. This significantly limited the number of sites available for the charge storage.

Recently, researchers have been looking at the deposition of nitrogen-containing conductive polymers, such as polyaniline and polypyrrole [8,43-46], on high surface area carbon after completing pyrolysis and activation processes. These polymers create Faradic pseudocapacitance by reversibly oxidizing and reducing over a potential range. Polyaniline is perhaps the most researched because it has several oxidation-state structures [47]. Adding this nitrogen-containing polymer to carbon has been found to increase the capacitance significantly due to the combined effects from double-layer capacitance and pseudocapacitance [8]. However, problems arise due to the large polymers blocking the carbon pores during deposition, decreasing the surface area available for charge storage. Additionally, swelling and shrinking of the polymers may lead to decreased cyclic stability due to mechanical breakdown $[8,48]$. Coating the pore surfaces of high surface area carbon with a smaller nitrogen-containing molecule may alleviate these issues.

In this paper, a simple solution-based method is used as a proof-of-concept, with the intention of eliminating the need for the complicated and/or expensive processing that is sometimes used to introduce nitrogen atoms into porous carbon structures. In order to achieve high capacitance contributions from both double layer formation and pseudocapacitive reactions, the porous carbon was modified after pyrolysis and activation steps to maintain a high specific surface area while achieving a high concentration of nitrogen. In addition, the influence of the location of the added nitrogen on material properties and electrochemical performance was investigated. Nitrogen either remains on the surface of highly porous carbon as functional groups or is incorporated into the bulk carbon network as heteroatoms. Both nitrogen-modified samples are then compared to the original unmodified porous carbon. Multiple techniques were used to analyze the samples, including nitrogen sorption analysis, X-ray photoelectron spectroscopy (XPS), cyclic voltammetry (CV), galvanic cycling, and elec- 
trochemical impedance spectroscopy (EIS).

\section{RESULTS}

\section{Structure and composition}

Isotherms and pore size distributions from nitrogen sorption are shown in Fig. 1 and the corresponding surface area, pore volume, and pore size are listed in Table 1. All samples show similarly shaped isotherms, with contributions from micropores, mesopores, and macropores. Unmodified carbon (UC) has the highest amount of micropores, resulting in this sample having the highest total surface area. For hexamine-coated carbon (HTMA-C), the total surface area is significantly lower than UC. This suggests that hexamine is successfully introduced into the porous carbon, coating the inner surfaces of larger pores and filling the smaller pores [35]. Upon heat treatment of HTMA-C to incorporate nitrogen into the bulk carbon network, the total surface area increases as seen for nitrogen-modified carbon (NC), but still remains lower than UC. Interestingly, the mesopore surface area and mesopore volume for NC become larger than the values for UC, which indicates the formation of new mesopores when carbon is heated in the presence of hexamine through an activation process [33]. However, the microporous surface area for NC is still lower than that of $\mathrm{UC}$, suggesting that some nitrogen is retained in the micropores. The mesopore size distributions in Fig. 1c show how the mesopore volume changes after surface modification with hexamine. For HTMA-C, nitrogen-modified carbon has a slightly lower mesopore volume than UC. However, upon heat treatment to obtain NC, the mesoporous volume is recovered and even surpasses that of UC. The nitrogen in the hexamine coating may react with the carbon when heated, etching away some of the material to open up the porous structure [43]. In the micropore region shown in Fig. 1d, the decrease in pore volume after hexamine mod-
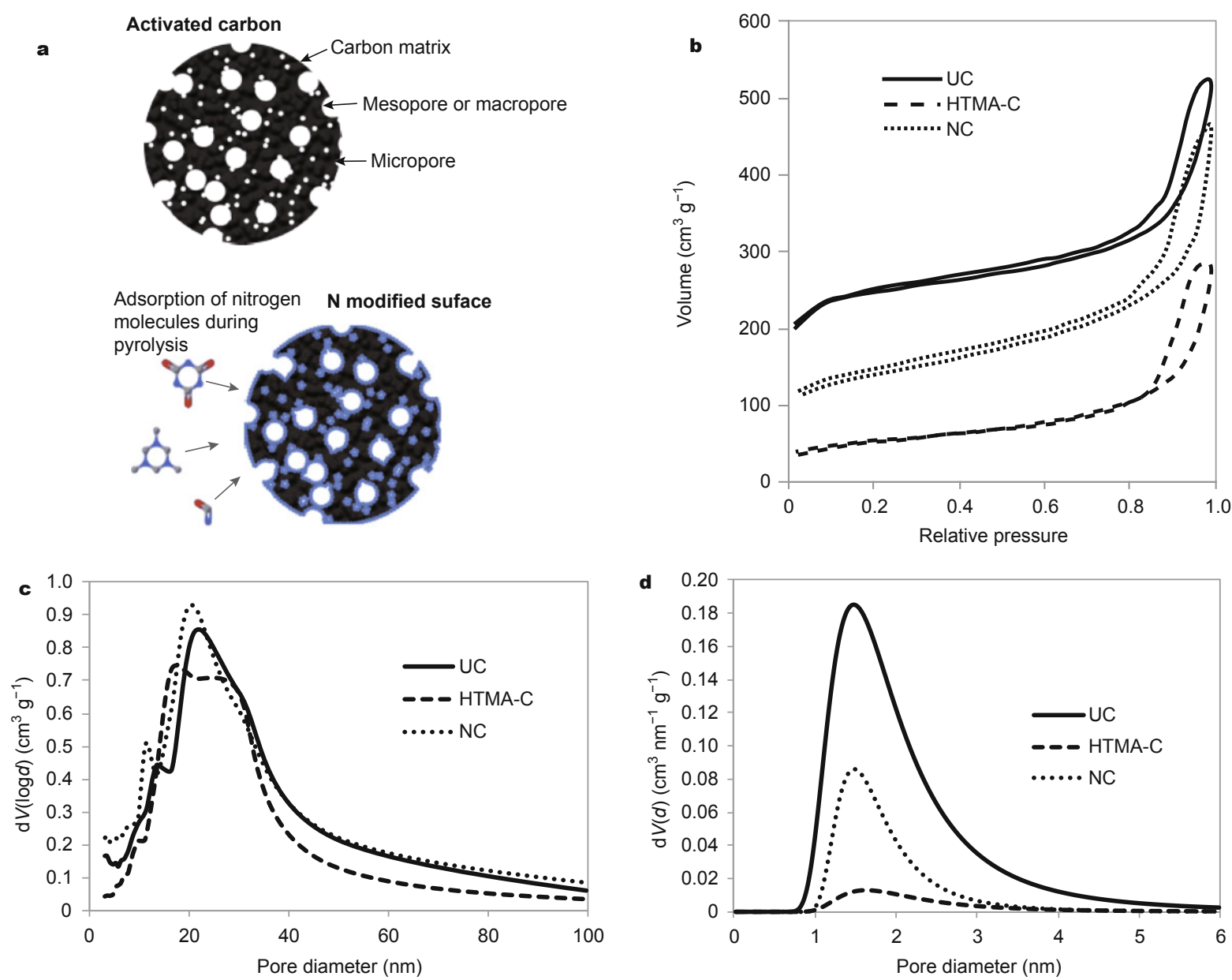

Figure 1 (a) Illustration of hexamine filling the carbon pores. (b) Nitrogen sorption isotherms, (c) mesopore size distribution, and (d) micropore size distribution for UC, HTMA-C, and NC. 
Table 1 Surface area, pore volume, and pore diameter from nitrogen sorption analysis. Contributions from both mesopores and micropores are listed

\begin{tabular}{|c|c|c|c|c|c|c|c|}
\hline \multirow{2}{*}{ Sample } & \multicolumn{3}{|c|}{ Surface area $\left(\mathrm{m}^{2} \mathrm{~g}^{-1}\right)$} & \multicolumn{2}{|c|}{ Pore volume $\left(\mathrm{cm}^{3} \mathrm{~g}^{-1}\right)$} & \multicolumn{2}{|c|}{ Pore diameter $(\mathrm{nm})$} \\
\hline & Total & Mesopore & Micropore & Mesopore & Micropore & Mesopore & Micropore \\
\hline UC & 735.7 & 134.3 & 550.4 & 0.46 & 0.30 & 3.2 & 1.4 \\
\hline HTMA-C & 172.4 & 96.4 & 34.6 & 0.39 & 0.02 & 3.7 & 1.6 \\
\hline $\mathrm{NC}$ & 442.8 & 167.8 & 188.4 & 0.53 & 0.11 & 3.2 & 1.5 \\
\hline
\end{tabular}

ification is much more significant. This suggests that the hexamine fills the micropores first, then partially fills the mesopores. An illustration of this mechanism is shown in Fig. 1a. Again, after heat treatment, some of the micropore volume is recovered. However, unlike in the mesoporous region, the micropore volume of $\mathrm{NC}$ remains lower than UC.

Table 2 lists the chemical compositions in wt.\% and at.\% for the three samples. All samples have a small amount of oxygen, which originates from the precursors and activation processes. No nitrogen is detected in UC even though nitrogen-containing hexamine was used as the reactive catalyst during synthesis. When heated above $850^{\circ} \mathrm{C}$, nitrogen is removed from carbon $[22,42]$, so it is likely that most of the nitrogen is removed during pyrolysis at $900^{\circ} \mathrm{C}$. As demonstrated with both HTMA-C and NC, modifying porous carbon with hexamine after pyrolysis and activation adds a fair amount of nitrogen. NC has more nitrogen than HTMA-C, which may be due to hexamine changing the behavior of porous carbon when heated at high temperature. Again, the hexamine in this sample may cause the removal of some carbon, leading to the higher nitrogen content in NC.

Survey spectra from XPS are shown in Fig. 2. UC shows large peaks for carbon and oxygen, as well as small peaks for nitrogen and fluorine. Nitrogen is present due to the use of hexamine as the reactive catalyst during cyrogel synthesis, although much of the nitrogen is lost during pyrolysis at high temperature $[22,42]$. The fluorine peak is from the polytetrafluoroethylene (PTFE) binder used to prepare samples for measurement and will not be analyzed further as it has minimal effect on the performance of the material. The modified carbon samples also show carbon and oxygen peaks, in addition to stronger nitrogen peaks. Al- though PTFE was also used to prepare these samples, the fluorine peak intensity is too low to separate from the background noise in the spectra. Each of the 3 samples exhibits a strong C1s peak near $284.8 \mathrm{eV}$, which is typical for pure carbon $[49,50]$. Detailed scans for O1s peak and N1s peak are shown in Fig. 3 and Fig. 4, respectively. A summary of each of the different peaks and their percent contribution is shown in Table 3. The detailed scans of O1s peak show that each of the three samples has a peak centering at approximately $533.1 \mathrm{eV}$ which can be attributed to oxygen single bonded to carbon in the form of phenol and/or ether groups [23,51]. A second peak is also present in each sample, centered around $531.5 \mathrm{eV}$, which may be attributed to oxygen double bonded to carbon as a quinone type group $[23,51]$. A very small third peak is seen at approximately $535.4 \mathrm{eV}$, which may correspond to chemisorbed oxygen or water $[23,51]$. After adding the hexamine coating to carbon, the relative amount of $\mathrm{C}-\mathrm{O}$ as compared to $\mathrm{C}=\mathrm{O}$ decreases. For $\mathrm{UC}$, the peak ratio of $\mathrm{C}-\mathrm{O}$ to $\mathrm{C}=\mathrm{O}$ is approximately $3: 2$, but this ratio decreases to $1: 1$ for $\mathrm{HT}$ $\mathrm{MA}-\mathrm{C}$. However, after heat treatment the ratio of $\mathrm{C}-\mathrm{O}$ to $\mathrm{C}=\mathrm{O}$ increases again, with a ratio of 3:2 for NC.

A very significant difference between HTMA-C and NC can be found when looking at the detailed scan for N1s peak in Fig. 4. HTMA-C shows one large peak at approximately $399.8 \mathrm{eV}$ and a much smaller peak at approximately $401.4 \mathrm{eV}$. NC shows two distinct peaks at $400.9 \mathrm{eV}$ and $398.9 \mathrm{eV}$. For HTMA-C, the peak at $399.8 \mathrm{eV}$ corresponds to amine or cyano groups $[38,50]$, while the smaller peak at $401.4 \mathrm{eV}$ corresponds to quaternary nitrogen (N-Q) $[22,25]$. The significantly larger intensity of the peak at $399.8 \mathrm{eV}$ suggests that most of the nitrogen found in this sample is in the form of surface funtionalities. For NC, the peak at $400.9 \mathrm{eV}$ corresponds to pyrrolic nitrogen (N-5)

Table 2 Chemical compositions for UC, HTMA-C, and NC from EDAX

\begin{tabular}{lcccccc}
\hline \multirow{2}{*}{ Sample } & \multicolumn{3}{c}{ Composition (wt.\%) } & \multicolumn{2}{c}{ Composition (at.\%) } \\
\cline { 2 - 6 } & $\mathrm{C}$ & $\mathrm{N}$ & $\mathrm{O}$ & $\mathrm{C}$ & $\mathrm{N}$ \\
\hline UC & 98.1 & - & 1.9 & 98.6 & - \\
HTMA-C & 90.5 & 8.0 & 1.5 & 91.9 & 7.4 \\
NC & 89.0 & 9.8 & 1.2 & 90.5 & 1.2 & 8.6 \\
\hline
\end{tabular}



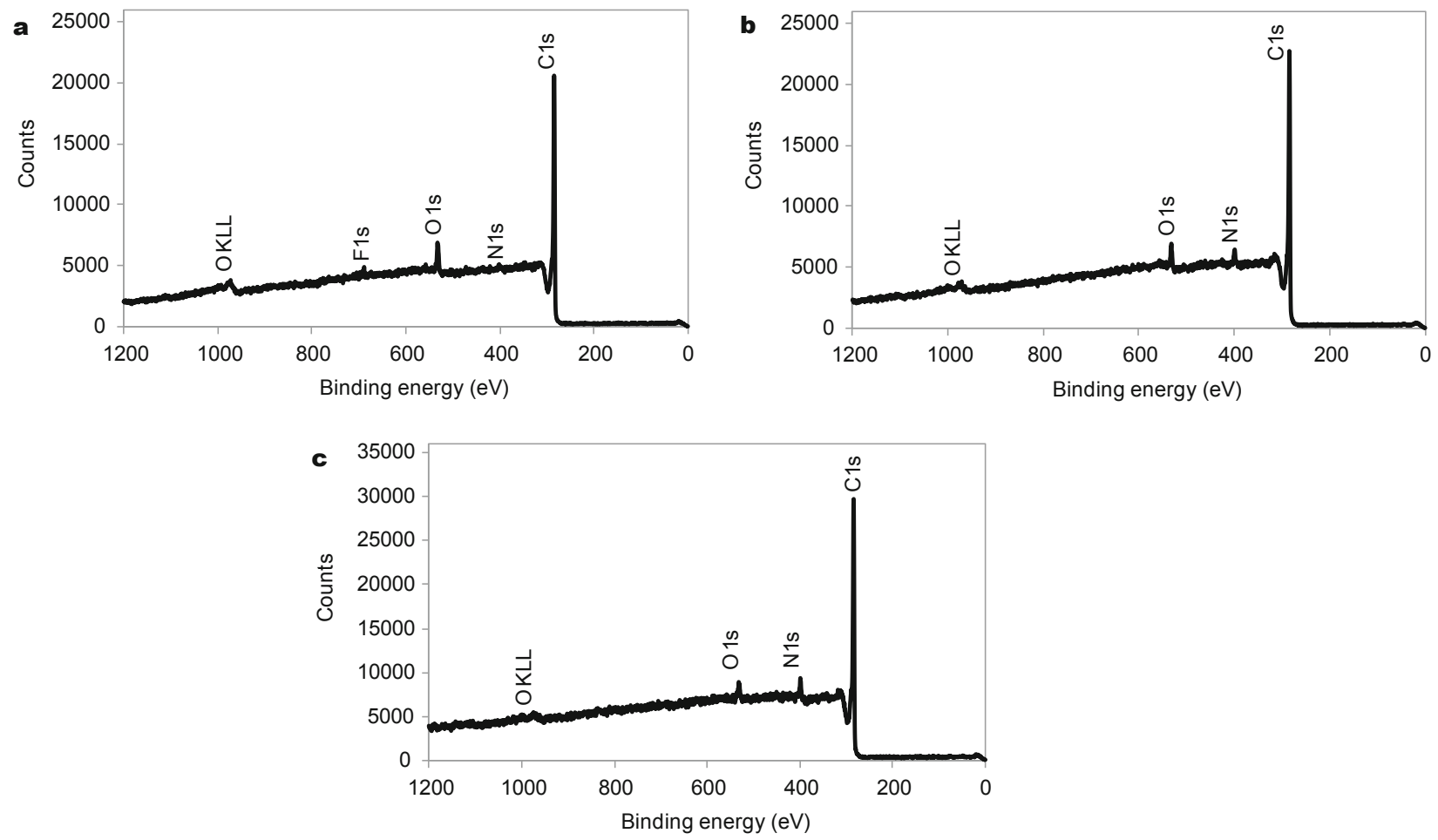

Figure 2 XPS survey scans for (a) UC, (b) HTMA-C, and (c) NC.
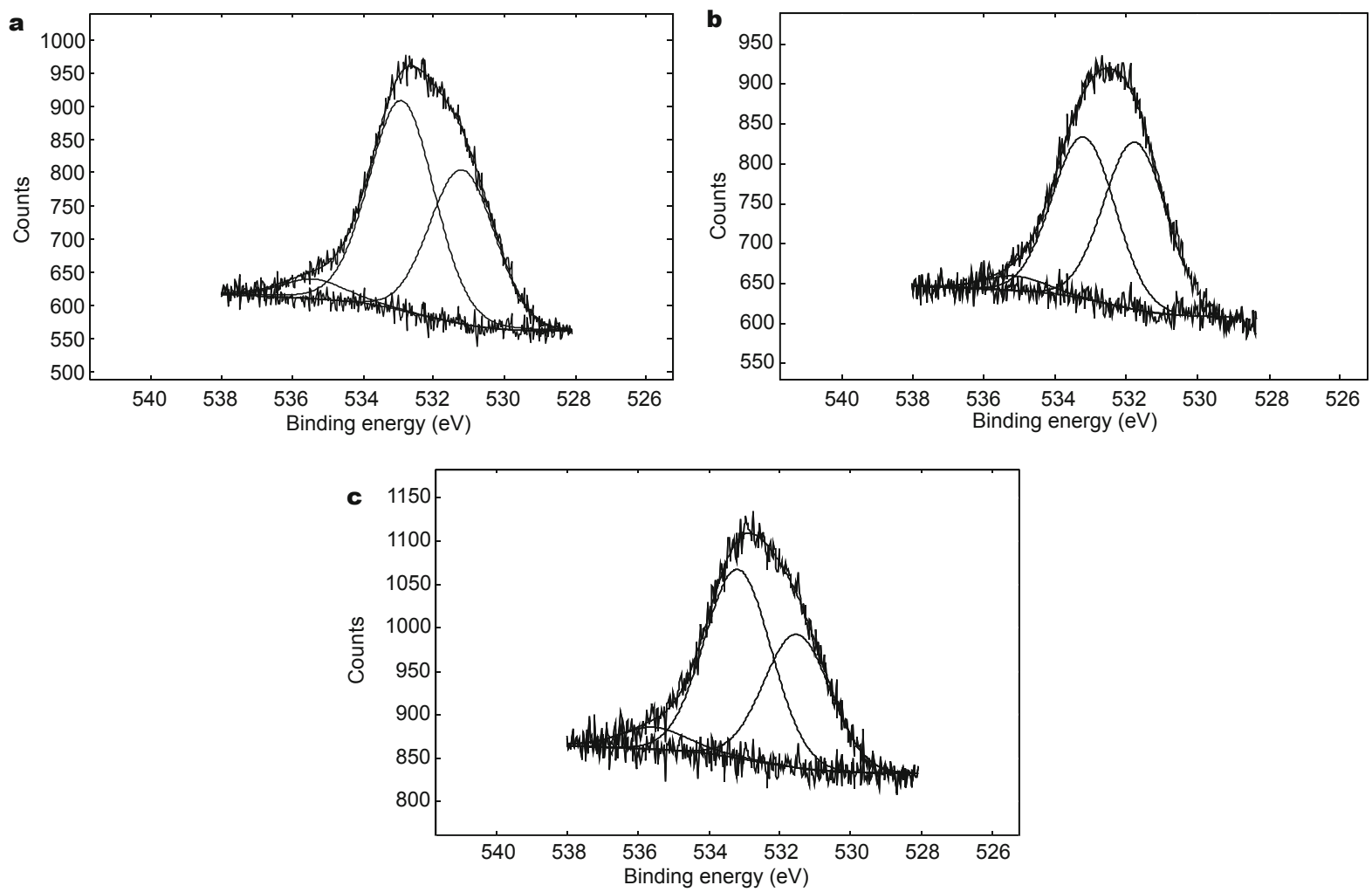

Figure 3 Detailed scans for the O1s peak for (a) UC, (b) HTMA-C, and (c) NC. 

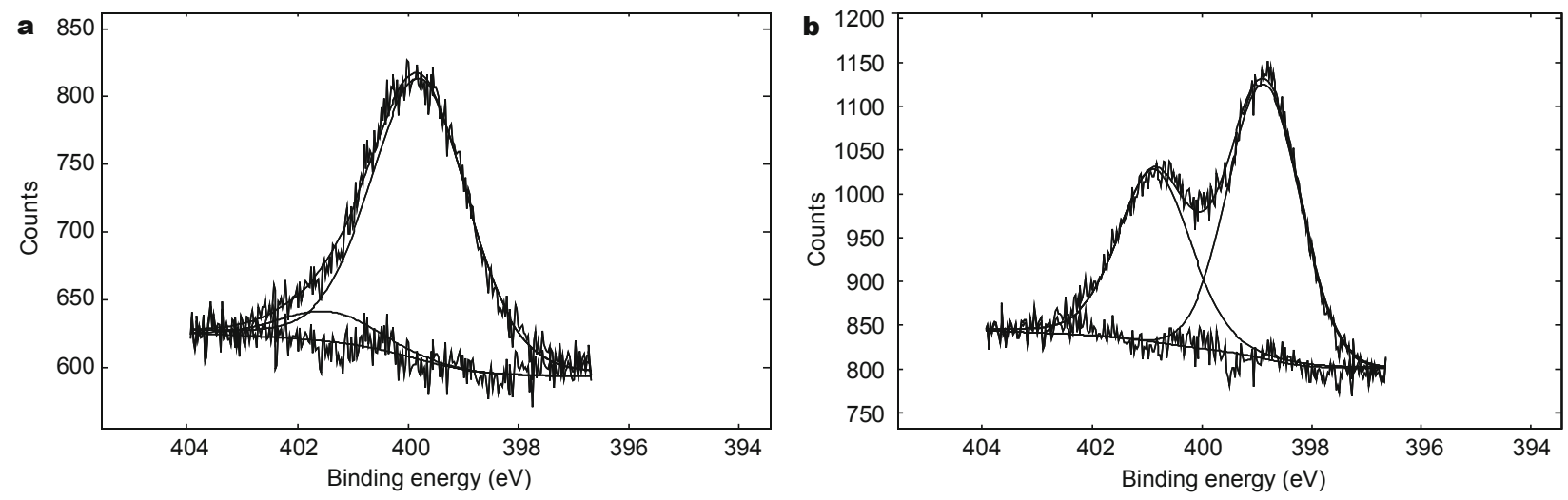

Figure 4 Detailed scans for the N1s peak for (a) HTMA-C and (b) NC.

Table 3 Percent contributions for the different functional groups as measured by XPS

\begin{tabular}{|c|c|c|c|c|c|c|c|}
\hline \multirow{3}{*}{ Sample } & \multicolumn{3}{|c|}{ O1s } & \multicolumn{4}{|c|}{ N1s } \\
\hline & $\mathrm{C}=\mathrm{O}$ & $\mathrm{C}-\mathrm{O}$ & Water & N-6 & Amine/cyano & $\mathrm{N}-5$ & N-Q \\
\hline & $531.5 \mathrm{eV}$ & $533.1 \mathrm{eV}$ & $535.4 \mathrm{eV}$ & $398.9 \mathrm{eV}$ & $399.8 \mathrm{eV}$ & $400.9 \mathrm{eV}$ & $401.1 \mathrm{eV}$ \\
\hline UC & 0.39 & 0.56 & 0.06 & - & - & - & - \\
\hline HTMA-C & 0.48 & 0.47 & 0.04 & - & 0.91 & - & 0.09 \\
\hline $\mathrm{NC}$ & 0.38 & 0.55 & 0.07 & 0.59 & - & 0.41 & - \\
\hline
\end{tabular}

$[25,27,38]$, while the peak at $398.9 \mathrm{eV}$ corresponds to pyridinic nitrogen (N-6) [22,25]. Therefore, while the vast majority of nitrogen groups in HTMA-C are in the form of surface functionalities or a surface-only coating of hexamine, the heat treatment applied to NC helps to incorporate nitrogen into carbon's bulk network in the form of substitutional heteroatoms. Such differences will have an effect on the electrochemical properties.

To investigate the effect of the different forms of nitrogen on the electrical conductivity of the carbon, resistance measurements were taken using a 4-point probe. UC has a resistance of approximately $5.18 \Omega$, while both of the samples containing nitrogen have lower resistances. For HTMA-C, the resistance is $4.01 \Omega$ and for NC is even lower at $2.57 \Omega$. For HTMA-C, the amine groups contribute lone pair electrons. In NC, the pyrrolic nitrogen contributes two p-electrons to the delocalized $\pi$-electron system, while pyridinic nitrogen contributes one p-electron to the $\pi$-electron system as well as lone electron pairs [38]. In these ways, modification helps to increase electronic conductivity through carbon.

\section{Electrochemical Analysis}

Cyclic voltammograms taken at 10,50 , and $100 \mathrm{mV} \mathrm{s}^{-1}$ and galvanic cycling curves taken at $0.5,1,5$, and $10 \mathrm{~mA}$ for each sample are shown in Fig. 5. Compared to unmodified carbon, there is some distortion in the CV curves for the modified samples. This suggests the possible presence of pseudocapacitance, although no redox peaks are clearly visible. The measured current is also higher for this sample than UC, suggesting higher capacitance. Due to the much lower surface area for HTMA-C as compared to UC, it can be assumed that the enhanced performance is from pseudocapacitive reactions induced by the hexamine coating. The CV for the NC sample shows a measured current that is much lower than that of HTMA-C. Surprisingly, it is also slightly lower than the measured current for UC. A small peak still occurs as the voltage approaches $2 \mathrm{~V}$, suggesting that some pseudocapacitive reactions are still present. From the GC curves, all samples show minimal voltage drops at the onset point of discharge. However, the differences in discharge time are significant. Mirroring the findings from the CV curves, HTMA-C shows the best performance as evidenced by the longest discharge time, while $\mathrm{NC}$ has the lowest performance.

Values for capacitance normalized to mass, total surface area, and volume are listed in Table 4. The gravimetric capacitance is highest for HTMA-C, increasing by almost $10 \%$ when compared to UC despite the added mass from the hexamine coating. Similarly, the volumetric ca- 

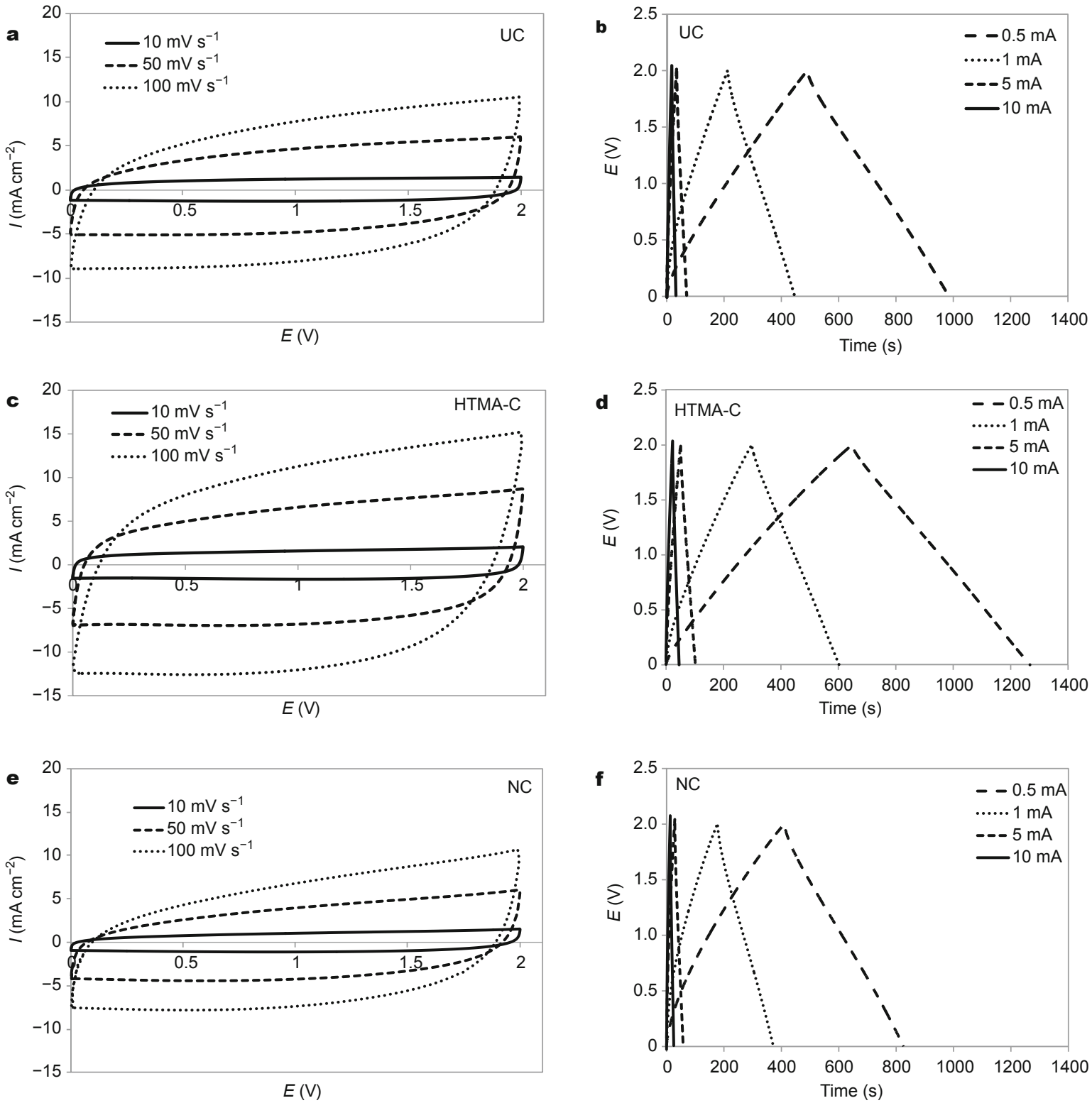

Figure 5 Cyclic voltammograms taken at 10,50 , and $100 \mathrm{mV} \mathrm{s}^{-1}$ (left) and galvanic cycles taken at $0.5,1,5$, and $10 \mathrm{~mA}$ (right) for UC (a and b), HTMA-C (c and d), and NC (e and f).

Table 4 Capacitance normalized to mass, total surface area, and volume

\begin{tabular}{lccc}
\hline Sample & Gravimetric capacitance $\left(\mathrm{F} \mathrm{g}^{-1}\right)$ & Capacitance normalized to total surface area $\left(\mathrm{F} \mathrm{m}^{-2}\right)$ & Volumetric capacitance $\left(\mathrm{F} \mathrm{cm}^{-3}\right)$ \\
\hline UC & 80.2 & 0.109 & 42.5 \\
HTMA-C & 87.8 & 0.509 & 49.8 \\
NC & 64.7 & 0.153 & 35.7 \\
\hline
\end{tabular}

pacitance is highest for HTMA-C. It should be noted that neither the specific surface area nor nitrogen content has been optimized to achieve the highest capacitance in these devices, resulting in the relatively modest capacitance val- ues. Alternatively, this is an investigation of the importance of nitrogen location in the porous carbon and its effect on the electrochemical performance. When the capacitance is normalized to total surface area, the effect of the hexamine 
coating is even more prominent. The capacitance is nearly 5 times higher for HTMA-C than UC, a direct result of modification from the hexamine coating. However, when nitrogen is incorporated into the bulk carbon network rather than remaining on the surface as a hexamine coating, it is detrimental to charge storage as evidenced by the significantly decreased capacitance for NC. Nyquist plots from EIS are shown in Fig. 6. For HTMA-C, the linear portion of the curve at lower frequencies has a more vertical slope, indicating that the diffusivity resistance is the lowest for this sample. The hexamine in HTMA-C may be smoothing the carbon pore surfaces, decreasing scattering effects and making it easier for the electrolyte ions to move in and out of the pores. However, the diffusivity resistance of NC increases and surpasses that of UC. Table 5 shows that the equivalent series resistance (ESR) increases slightly for carbon after modification for both NC and HTMA-C, but there is very little difference between the two samples. However, a difference can be seen for the $R_{\mathrm{CT}}$, increasing by almost $50 \%$ for NC. Since the starting nitrogen concentration is lower for HTMA-C, it can be assumed that this disparity is due to the dissimilar locations of nitrogen in each of the samples. The incorporation of nitrogen into the bulk network, rather than remaining in the form of surface functional groups or a surface coating, is non-ideal for supercapacitors and results in decreased performance. Furthermore, $R_{\mathrm{CT}}$ is lower for HTMA-C than for UC. This suggests that the hexamine coating facilitates better charge transfer at the electrode/electrolyte interface likely due to
Table 5 ESR and charge transfer resistance $\left(R_{\mathrm{CT}}\right)$ for UC, HTMA-C, and $\mathrm{NC}$

\begin{tabular}{ccc}
\hline Sample & $\operatorname{ESR}(\Omega)$ & $R_{\mathrm{CT}}(\Omega)$ \\
\hline UC & 1.92 & 3.79 \\
HTMA-C & 2.18 & 3.06 \\
NC & 2.16 & 4.47 \\
\hline
\end{tabular}

enhanced surface charge density, which then helps to significantly improve performance of this material for supercapacitors.

To test the effect of lower $R_{\mathrm{CT}}$, the samples were cycled at higher applied currents during galvanic cycling. With a low $R_{\mathrm{CT}}$, the samples will be able to react to the changing charge and discharge current faster, resulting in higher capacitance. Fig. 7a shows how the gravimetric capacitance varies with the applied current for all three samples. At the lowest applied current of $0.5 \mathrm{~mA}$, the gravimetric capacitance for HTMA-C is almost $10 \%$ higher than that for UC. At a higher applied current of $10 \mathrm{~mA}$, HTMA-C has a capacitance that is over $35 \%$ higher than UC. These results suggest that careful coating of carbon with nitrogen-containing hexamine is very beneficial for enhancing the performance of supercapacitors, especially those subjected to high charge and discharge rates.

Finally, all three samples were cycled at charging rates ranging from 0.5 to $10 \mathrm{~mA}$ to test the stability of each material. The results are shown in Fig. 7b. Each sample experiences a capacitance drop within the first 100 cycles,
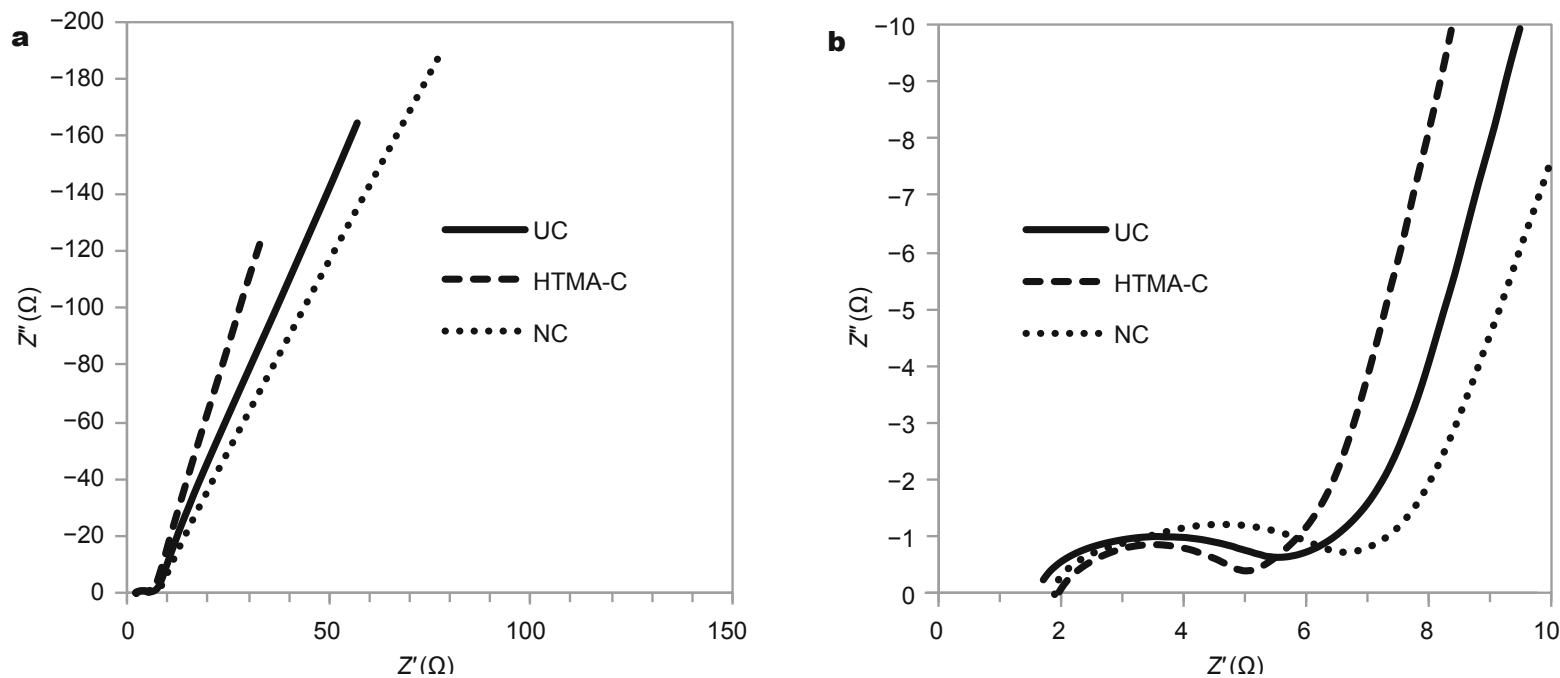

Figure 6 Nyquist plots (a) over the entire frequency range and (b) at high frequency only. 

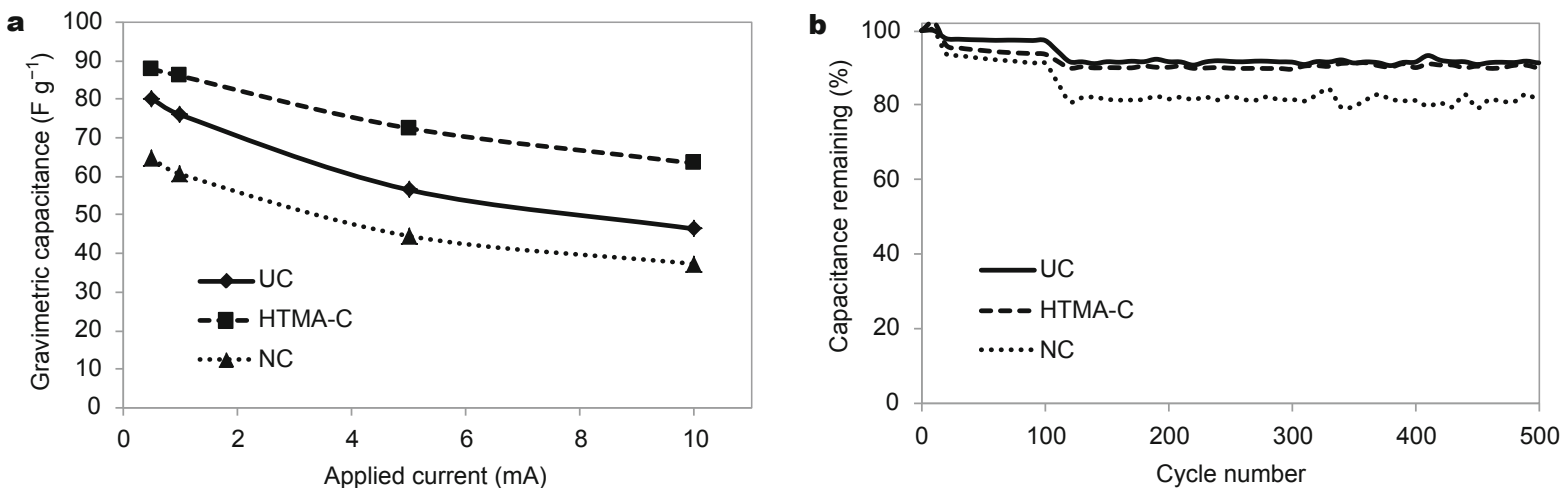

Figure 7 (a) Gravimetric capacitance at various applied currents for UC, HTMA-C, and NC. Carbon coated with hexamine consistantly shows the highest capacitance, especially at high applied currents. (b) Cyclic stability for UC, HTMA-C, and NC taken at various applied currents.

which may be a result of a small amount of impurities such as oxygen in the electrode material or water in the electrolyte causing irreversible reactions. The slight drop in capacitance at 100 cycles is the result of the testing conditions, as each sample was subjected to electrochemical impedance spectroscopy measurements at this point. The NC sample loses the largest fraction of its initial capacitance during the first 100 cycles. This sample also appears to have the most variability in capacitance after 100 cycles. However, for both UC and HTMA-C, the capacitance is nearly unchanged after the first 100 cycles, each sample decreasing by less than $0.5 \%$ after 500 cycles. This suggests that the hexamine coating is fairly stable and does not decompose significantly during initial cycling. Additionally, unlike large polymer coatings, the hexamine coating does not appear to swell or shrink during cycling, so the cyclic stability does not decrease due to mechanical breakdown $[8,48]$.

\section{DISCUSSION}

Nitrogen-containing hexamine can be coated onto the surface of highly porous carbon using a simple solution-based method in order to induce pseudocapacitive reactions in the supercapacitors utilizing organic electrolyte. Upon heat treatment, the hexamine coating decomposes and nitrogen is incorporated into the bulk carbon network as substituted heteroatoms. The addition of nitrogen both on the surface and in the bulk leads to decreased electrical resistance in the carbon material. Electrochemical measurements show that the hexamine surface coating on porous carbon can increase capacitance, despite a significant decrease in the surface area available for charge storage. It has been shown that the form and location of nitrogen in the porous carbon is very important and leads to different material properties. When nitrogen is incorporated into bulk network and is no longer restricted to the surface, the capacitance is lower than that when nitrogen remains in the form of surface functional groups. From XPS, it is seen that the form of nitrogen in NC is different from HTMA-C, converting from surface functional groups in HTMA-C to heteroatoms incorporated into the bulk carbon network in NC. The amine and/or cyano functional groups in HTMA-C have lone pair electrons, which increase the surface charge density. While NC does contain negatively charged nitrogen groups in the form of pyrrolic and pyridinic nitrogen that can increase conductivity and add pseudocapacitance $[27,52]$, these charges are distributed throughout the bulk rather than remaining only on the surface and therefore do not interact as directly with the electrolyte. Fig. 8 illustrates the difference in the charge distributions between these two samples and helps to explain the higher capacitance for HTMA-C compared to NC (personal communication with Skinner B). For HTMA-C, the increased charge density

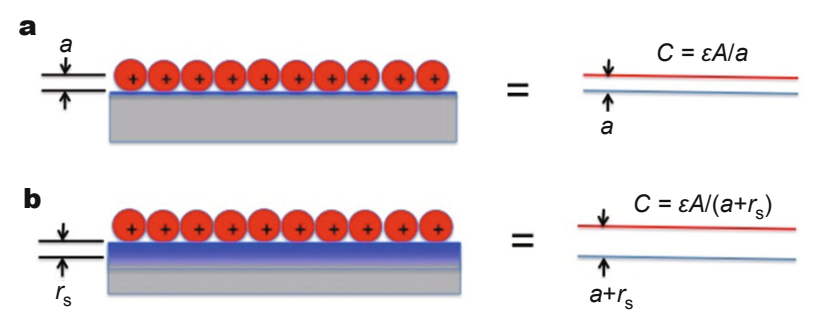

Figure 8 Different charge distributions for each nitrogen-modified carbon lead to different capacitance values for each sample. (a) Nitrogen remains in the form of functional groups that increase the charge density at the surface for HTMA-C, leading to a small charge separation distance $a$ and higher capacitance. (b) Upon heat treatment for NC, nitrogen is incorporated into the bulk carbon network and is no longer restricted to the surface, leading to additional charge separation distance $r_{\mathrm{s}}$ and lower capacitance. 
from nitrogen modification is restricted at the surface of the porous carbon. The charge separation distance is very small, which leads to higher capacitance. For NC, nitrogen groups are incorporated into the bulk, spreading the additional charges over a greater distance. This leads to a larger charge separation distance and a lower capacitance as a result. Therefore, despite the higher nitrogen content in NC, the advantageous location of nitrogen in surface functional groups in the hexamine coating for HTMA-C, rather than being in the bulk network, leads to this sample's superior capacitance. HTMA-C also has a low diffusivity resistance and charge transfer resistance, resulting in enhanced performance at high charge and discharge rates. Additionally, the cyclic stability is comparable to UC. This research can be used as a starting point for further investigations, as it is believed that the simple solution method for modification presented here can be easily performed with a variety of electrode materials and dopants and with careful optimization can lead to greatly enhanced supercapacitors.

\section{EXPERIMENTAL SECTION}

\section{Synthesis}

Highly porous carbon cryogels were prepared from precursors resorcinol and furaldehyde using hexamethylenetetramine (hexamine or HTMA) as the catalyst. The procedure for synthesizing highly porous carbon was similar to previous reports $[53,54]$. The furaldehyde to resorcinol molar ratio was set to 2.5 and the resorcinol to hexamine molar ratio is 75 . The resorcinol and furaldehyde were mixed together first, followed by the addition of the solvent tert-butanol. Hexamine was added last as the reactive catalyst. The mixture was sealed and cured in an oven at $80^{\circ} \mathrm{C}$ for 7 days to allow for gelation and aging to strengthen the newly-formed three-dimensional gel network. The wet organic gel is dried under vacuum at $-50^{\circ} \mathrm{C}$ in a Labconco FreeZone $1 \mathrm{~L}$ freeze dryer. After drying for several days, the organic gel was pyrolyzed at $900^{\circ} \mathrm{C}$ for $3 \mathrm{~h}$ under nitrogen flow with a ramp rate of $5^{\circ} \mathrm{C} \mathrm{min}^{-1}$ to obtain the porous carbon cryogel. Finally, physical activation was performed in the presence of oxygen in order to etch away a controlled amount of loosely-bound material and open up the porous structure. Here, activation took place under dry air flow at $420^{\circ} \mathrm{C}$ for $4 \mathrm{~h}$ with a ramp rate of $5^{\circ} \mathrm{C} \mathrm{min}^{-1}$, followed by heating again $900^{\circ} \mathrm{C}$ for $3 \mathrm{~h}$ under nitrogen flow to remove new functional groups that might have formed during activation. The activation dwell time was selected in order to obtain a mass loss of approximately $50 \%$ [55].

To surface modify carbon with hexamine, a simple solution method was used, which was adapted from our previous reports [41,42]. Specifically, a solution of 4 wt.\% hexamine in tert-butanol was stirred and heated at $80^{\circ} \mathrm{C}$ for several days. Both lower (2 wt.\%) and higher (6 wt.\%) hexamine concentrations were investigated in preliminary experiments, but resulted in lower nitrogen concentrations in the porous carbon ( $2 \mathrm{wt} . \%)$ or significant pore blockage (6 wt.\%). Next, large pieces of the activated carbon cryogels were soaked in the hexamine solution at $80^{\circ} \mathrm{C}$ for three days. The hexamine modified samples were then freeze dried to remove any remaining solvent. This process resulted in a hexamine coating on the surface of the carbon pores. This sample was labelled as HTMA-C. To create nitrogen-modified carbon, HTMA-C samples were heated at $500^{\circ} \mathrm{C}$ for $3 \mathrm{~h}$ under nitrogen flow to decompose the hexamine coating and incorporate nitrogen heteroatoms into the bulk network of the porous carbon. No hexamine remained on the surface of the carbon pores. This nitrogen-modified carbon sample was labelled as NC. Unmodified carbon was labelled as UC.

\section{Characterization}

Nitrogen sorption isotherms were measured using a Quantachrome NOVA 4200e. Samples were degassed under vacuum at elevated temperature for at least $6 \mathrm{~h}$ prior to the measurement. The multipoint Brunauer-Emmett-Teller (BET) method was used to determine the total surface area. For the mesopore surface area, pore volume, and pore diameter, the Barrett-Joyner-Halenda (BJH) method was used. Micropore surface area and pore volume were determined using the t-method, and the micropore diameter was determined using the Dubinin-Astakhov (DA) method. Atomic compositions of the samples were taken using a FEI Sirion SEM operating at $5 \mathrm{kV}$ equipped with energy-dispersive X-ray spectroscopy (EDAX). Samples were sputter coated with $\mathrm{Au} / \mathrm{Pd}$ prior to measurements. XPS spectra were taken on a PHI 5000 VersaProbe setup using Al Ka radiation. Data analysis was carried out using PHI MultiPak software (Physical Electronics, Chanhassen, MN).

\section{Electrochemical analysis}

Electrodes for electrochemical measurements were prepared from UC, HTMA-C, or NC by first grinding the large pieces into a fine powder. Approximately 3 wt.\% of PTFE was added to the powder as a binder. The mixture was mechanically rolled into sheets with a thickness of $0.07-0.08$ $\mathrm{mm}$ and electrodes were punched out with a diameter of $10 \mathrm{~mm}$. A Celgard ${ }^{\circledast}$ porous film separating the electrodes to prevent short circuiting and carbon coated aluminum contacts were used as current collectors. The two-electrode assembly was pressed under vacuum overnight to ensure 
good contact between the electrodes and the current collectors and to remove moisture from the porous electrodes. The electrolyte used was $1 \mathrm{M}$ tetraethylammonium tetrafluoroborate (TEATFB) in 1:1 propylene carbonate:dimethylcarbonate. The electrode assembly was placed in a flat cell and electrolyte was added in an argon-filled glovebox. The samples were placed under vacuum to increase the penetration of the electrolyte into the pores. To avoid nitrogen-induced pseudocapacitive reactions in both electrodes, all samples were prepared with UC as the counter and reference electrode [18].

$\mathrm{CVs}$ and galvanic cycles (GCs) were taken using a Solatron $1287 \mathrm{~A}$ with a voltage range between 0 and $2 \mathrm{~V}$. The CVs were measured at scan rates of 100,50 , and $10 \mathrm{mV}$ $\mathrm{s}^{-1}$ and the GCs at 100, 50, 10, 5, 1, and $0.5 \mathrm{~mA}$. EIS was performed using the Solartron 1287A in conjunction with a Solartron 1260FRA/impedance analyzer. Measurements took place after performing $\mathrm{CV}$ and galvanic cycling at all the different rates listed above. The samples were pretreated for $10 \mathrm{~min}$ at $2 \mathrm{~V}$, and an AC voltage amplitude of $10 \mathrm{mV}$ and frequency range of $0.1 \mathrm{MHz}-1 \mathrm{mHz}$ were used for this scan. Cyclic stability tests were performed after completing the CVs, GCs, and EIS measurements. Samples were subjected to repeated charge and discharge during galvanic cycling at various rates between 1 and $10 \mathrm{~mA}$ up to 500 cycles.

The specific capacitance in $\mathrm{F} \mathrm{g}^{-1}$ was calculated from the discharge slope during galvanic cycling according to the following equation:

$$
C=\frac{4 I \Delta t}{\Delta V m}
$$

where $I$ is the discharge current in A, $\Delta t$ is the discharge time in $s, \Delta V$ is the discharge voltage in $\mathrm{V}$, and $m$ is the total mass of the active materials in both electrodes given in $\mathrm{g}$ [56]. Capacitance based on total surface area in $\mathrm{F} \mathrm{m}^{-2}$ was calculated by dividing the specific capacitance by the total surface area of the working electrode material found using nitrogen sorption analysis. Volumetric capacitance in $\mathrm{F} \mathrm{cm}^{-3}$ was calculated by multiplying the specific capacitance by the total density of both electrodes.

\section{Received 26 May 2015; accepted 23 June 2015;} published online 15 July 2015

1 Hall PJ, Mirzaeian M, Fletcher SI, et al. Energy storage in electrochemical capacitors: designing functional materials to improve performance. Energ Environ Sci, 2010, 3: 1238-1251

2 Liu C, Li F, Ma LP, et al. Advanced materials for energy storage. Adv Mater, 2010, 22: E28-E62

3 Arico AS, Bruce P, Scrosati B, et al. Nanostructured materials for advanced energy conversion and storage devices. Nat Mater, 2005, 4: $366-377$
4 Zhang QF, Uchaker E, Candelaria SL, et al. Nanomaterials for energy conversion and storage. Chem Soc Rev, 2013, 42: 3127-3171

5 Candelaria SL, Shao YY, Zhou W, et al. Nanostructured carbon for energy storage and conversion. Nano Energ, 2012, 1: 195-220

6 Simon P, Gogotsi Y, Dunn B. Where do batteries end and supercapacitors begin. Science, 2014, 343: 1210-1211

7 Simon P, Gogotsi Y. Materials for electrochemical capacitors. Nat Mater, 2008, 7: 845-854

8 Chen WC, Wen TC, Teng HS. Polyaniline-deposited porous carbon electrode for supercapacitor. Electrochim Acta, 2003, 48: 641-649

9 Inagaki M, Konno H, Tanaike O. Carbon materials for electrochemical capacitors. J Power Sources, 2010, 195: 7880-7903

10 Barbieri O, Hahn M, Herzog A, et al. Capacitance limits of high surface area activated carbons for double layer capacitors. Carbon, 2005, 43: 1303-1310

11 Biener J, Stadermann M, Suss M, et al. Advanced carbon aerogels for energy applications. Energ Environ Sci, 2011, 4: 656-667

12 Dutta S, Bhaumik A, Wu KCW. Hierarchically porous carbon derived from polymers and biomass: effect of interconnected pores on energy applications. Energ Environ Sci, 2014, 7: 3574-3592

13 Frackowiak E. Carbon materials for supercapacitor application. Phys Chem Chem Phys, 2007, 9: 1774-1785

14 Paraknowitsch JP, Thomas A. Doping carbons beyond nitrogen: an overview of advanced heteroatom doped carbons with boron, sulphur, and phosphorus for energy applications. Energ Environ Sci, 2013, 6: 2839-2855

15 Wood KN, O'Hayre R, Pylypenko S. Recent progress on nitrogen/ carbon structures designed for use in energy and sustainability applications. Energ Environ Sci, 2014, 7: 1212-1249

16 Wang DW, Li F, Chen ZG, et al. Synthesis and electrochemical property of boron-doped mesoporous carbon in supercapacitor. Chem Mater, 2008, 20: 7195-7200

17 Zhao Y, Candelaria SL, Liu Q, et al. Porous carbon with high capacitance and graphitization through controlled addition and removal of sulfur-containing compounds. Nano Energ, 2015, 12: 567-577

18 Jurewicz K, Babel K, Ziolkowski A, et al. Ammoxidation of active carbons for improvement of supercapacitor characteristics. Electrochim Acta, 2003, 48: 1491-1498

19 Azais P, Duclaux L, Florian P, et al. Causes of supercapacitors ageing in organic electrolyte. J Power Sources, 2007, 171: 1046-1053

20 Frackowiak E, Beguin F. Carbon materials for the electrochemical storage of energy in capacitors. Carbon, 2001, 39: 937-950

21 Beguin F, Frackowiak E (eds.). Carbons for Electrochemical Energy Storage and Conversion Systems. Boca Raton: CRC Press, 2009

22 Hulicova D, Yamashita J, Soneda Y, et al. Supercapacitors prepared from melamine-based carbon. Chem Mater, 2005, 17: 1241-1247

23 Ania CO, Khomenko V, Raymundo-Pinero E, et al. The large electrochemical capacitance of microporous doped carbon obtained by using a zeolite template. Adv Funct Mater, 2007, 17: 1828-1836

24 Li WR, Chen DH, Li Z, et al. Nitrogen enriched mesoporous carbon spheres obtained by a facile method and its application for electrochemical capacitor. Electrochem Commun, 2007, 9: 569-573

25 Lota G, Grzyb B, Machnikowska H, et al. Effect of nitrogen in carbon electrode on the supercapacitor performance. Chem Phys Lett, 2005, 404: 53-58

26 Beguin F, Szostak K, Lota G, et al. A self-supporting electrode for supercapacitors prepared by one-step pyrolysis of carbon nanotube/ polyacrylonitrile blends. Adv Mater, 2005, 17: 2380-2384

27 Hulicova-Jurcakova D, Kodama M, Shiraishi S, et al. Nitrogenenriched nonporous carbon electrodes with extraordinary supercapacitance. Adv Funct Mater, 2009, 19: 1800-1809

28 Frackowiak E. Supercapacitors based on carbon materials and ionic liquids. J Braz Chem Soc, 2006, 17: 1074-1082 
$29 \mathrm{Li} \mathrm{M}$, Xue JM. Integrated synthesis of nitrogen-doped mesoporous carbon from melamine resins with superior performance in supercapacitors. J Phys Chem C, 2014, 118: 2507-2517

30 Qiu B, Pan CT, Qian WJ, et al. Nitrogen-doped mesoporous carbons originated from ionic liquids as electrode materials for supercapacitors. J Mater Chem A, 2013, 1: 6373-6378

31 Khalid B, Meng QH, Li JT, et al. Nitrogen rich graphene-crosslinked melamine formaldehyde carbon cryogels for supercapacitors. Electrochim Acta, 2014, 142: 101-107

32 Su FB, Poh CK, Chen JS, et al. Nitrogen-containing microporous carbon nanospheres with improved capacitive properties. Energ Environ Sci, 2011, 4: 717-724

33 Laheaar A, Delpeux-Ouldriane S, Lust E, et al. Ammonia treatment of activated carbon powders for supercapacitor electrode application. J Electrochem Soc, 2014, 161: A568-A575

34 Kawaguchi M, Itoh A, Yagi S, et al. Preparation and characterization of carbonaceous materials containing nitrogen as electrochemical capacitor. J Power Sources, 2007, 172: 481-486

35 Garcia BB, Candelaria SL, Cao GZ. Nitrogenated porous carbon electrodes for supercapacitors. J Mater Sci, 2012, 47: 5996-6004

36 Chen LF, Zhang XD, Liang HW, et al. Synthesis of nitrogen-doped porous carbon nanofibers as an efficient electrode material for supercapacitors. ACS Nano, 2012, 6: 7092-7102

37 Zhao L, Fan LZ, Zhou MQ, et al. Nitrogen-containing hydrothermal carbons with superior performance in supercapacitors. Adv Mater, 2010, 22: 5202-5206

38 Pels JR, Kapteijn F, Moulijn JA, et al. Evolution of nitrogen functionalities in carbonaceous materials during pyrolysis. Carbon, 1995, 33: 1641-1653

39 Kapteijn F, Moulijn JA, Matzner S, et al. The development of nitrogen functionality in model chars during gasification in $\mathrm{CO}_{2}$ and $\mathrm{O}_{2}$. Carbon, 1999, 37: 1143-1150

40 Jung MJ, Jeong E, Cho S, et al. Effects of surface chemical properties of activated carbon modified by amino-fluorination for electric double-layer capacitor. J Colloid Interf Sci, 2012, 381: 152-157

41 Sepehri S, Garcia BB, Zhang Q, et al. Enhanced electrochemical and structural properties of carbon cryogels by surface chemistry alteration with boron and nitrogen. Carbon, 2009, 47: 1436-1443

42 Candelaria SL, Garcia BB, Liu DW, et al. Nitrogen modification of highly porous carbon for improved supercapacitor performance. J Mater Chem, 2012, 22: 9884-9889

43 Wu MQ, Snook GA, Gupta V, et al. Electrochemical fabrication and capacitance of composite films of carbon nanotubes and polyaniline. J Mater Chem, 2005, 15: 2297-2303

44 Ingram MD, Pappin AJ, Delalande F, et al. Development of electrochemical capacitors incorporating processable polymer gel electrolytes. Electrochim Acta, 1998, 43: 1601-1605

45 Hughes M, Chen GZ, Shaffer MSP, et al. Electrochemical capacitance of a nanoporous composite of carbon nanotubes and polypyrrole. Chem Mater, 2002, 14: 1610-1613

46 Zhang K, Zhang LL, Zhao XS, et al. Graphene/polyaniline nanofiber composites as supercapacitor electrodes. Chem Mater, 2010,

\section{2: $1392-1401$}

47 Huang WS, Humphrey BD, Macdiarmid AG. Polyaniline, a novel conducting polymer-morphology and chemistry of its oxidation and reduction in aqueous-electrolytes. J Chem Soc Farad T 1, 1986, 82: 2385-2400

48 Kotz R, Carlen M. Principles and applications of electrochemical capacitors. Electrochim Acta, 2000, 45: 2483-2498

49 Sanchez-Lopez JC, Donnet C, Lefebvre F, et al. Bonding structure in amorphous carbon nitride: a spectroscopic and nuclear magnetic resonance study. J Appl Phys, 2001, 90: 675-681

50 Dementjev AP, de Graaf A, van de Sanden MCM, et al. X-ray photoelectron spectroscopy reference data for identification of the $\mathrm{C}_{3} \mathrm{~N}_{4}$ phase in carbon-nitrogen films. Diam Relat Mater, 2000, 9: 1904-1907

51 Hulicova-Jurcakova D, Seredych M, Lu GQ, et al. Combined effect of nitrogen- and oxygen-containing functional groups of microporous activated carbon on its electrochemical performance in supercapacitors. Adv Funct Mater, 2009, 19: 438-447

52 Seredych M, Hulicova-Jurcakova D, Lu GQ, et al. Surface functional groups of carbons and the effects of their chemical character, density and accessibility to ions on electrochemical performance. Carbon, 2008, 46: 1475-1488

53 Wu DC, Fu RW, Zhang ST, et al. The preparation of carbon aerogels based upon the gelation of resorcinol-furfural in isopropanol with organic base catalyst. J Non-Cryst Solids, 2004, 336: 26-31

54 Garcia BB, Liu DW, Sepehri S, et al. Hexamethylenetetramine multiple catalysis as a porosity and pore size modifier in carbon cryogels. J Non-Cryst Solids, 2010, 356: 1620-1625

55 Feaver A, Cao GZ. Activated carbon cryogels for low pressure methane storage. Carbon, 2006, 44: 590-593

56 Lei Z, Chen Z, Zhao XS. Growth of polyaniline on hollow carbon spheres for enhancing electrocapacitance. J Phys Chem C, 2010, 114: $19867-19874$

Acknowledgements The authors greatly appreciate the help of several researchers at the University of Washington, in particular Chris Dandeneau, Yi Hsun Yang, and Prof. Fumio Ohuchi for XPS measurements. This work was funded by US National Science Foundation (NSF) (CMMI-1030048), the University of Washington's IGERT: Bioresource-based Energy for Sustainable Societies (DGE-0654252), and the Intel Corporation. Part of this work was conducted at the University of Washington NanoTech User Facility, a member of the NSF National Nanotechnology Infrastructure Network (NNIN).

Author contributions Candelaria SL synthesized and modified all samples, performed electrochemistry and data analysis, and wrote this manuscript. Uchaker E performed SEM, EDAX, and provided valuable discussion on electrochemistry results. Cao $G$ provided suggestions, helped interpret experimental results, and assisted in the preparation and revision of this manuscript.

Conflict of interest The authors declare that they have no conflict of interest. 


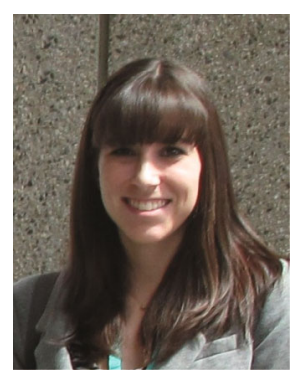

Stephanie Candelaria received her $\mathrm{PhD}$ degree in materials science and engineering at the University of Washington under the supervision of Prof. Guozhong Cao. Her research focused on sol-gel processing and electrochemical characterization of highly porous carbon from renewable resources for supercapacitors, with particular emphasis on surface modification for improved performance.

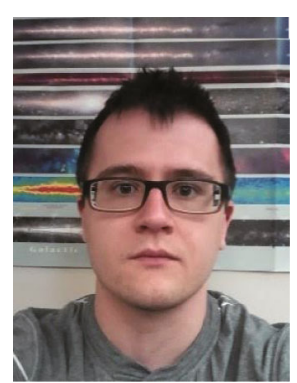

Evan Uchaker received his $\mathrm{PhD}$ degree in materials science and engineering at the University of Washington under the supervision of Prof. Guozhong Cao. His research interests are focused on the development and understanding of kinetically stabilized and defected electrode materials for electrochemical energy storage devices such as alkali-ion batteries.

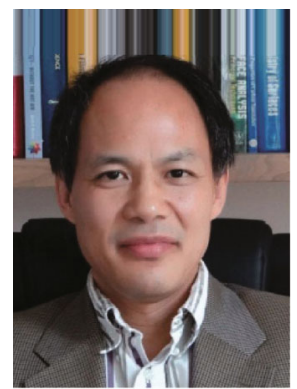

Guozhong Cao is Boeing-Steiner Professor of materials science and engineering, professor of chemical engineering, and adjunct professor of mechanical engineering at the University of Washington, and also a professor at Beijing Institute of Nanoenergy and Nanosystems, Chinese Academy of Sciences and Dalian University of Technology. His current research is focused on chemical processing of nanomaterials for energy related applications including solar cells, rechargeable batteries, supercapacitors, and hydrogen storage.

中文摘要 溶胶-凝胶法制备的高孔隙度多孔碳材料可通过简单溶液法用氮进行改性从而增加电容. 氮的引入可采用表面氮改性通过 引入六亚甲基四胺涂层再热解分解, 或在碳网结构中引入氮两种方式. 本文对两种材料的性能和电化学行为进行测量并与未改性的多 孔碳进行比较. 在碳网结构中的氮表现出增强的导电性, 而表面进行氮改性的多孔碳对于增强电容更有效. 氮改性还可以通过减少扩 散电阻和电荷转移电阻, 进而增强超级电容器在高倍率的充放电性能. 